Departamento de Historia

Universidad de Santiago de Chile

Revista de Historia Social

y de las Mentalidades

Volumen 23, $\mathrm{N}^{\circ} 1,2019: 189-210$

Issn: 0717-5248

Issn On Line: 0719-4749

\title{
INTELECTUALES Y NUEVA DERECHA EN ARGENTINA: EXPERTOS, MEDIÁTICOS E IDEÓLOGOS EN LA CONSTITUCIÓN DEL PRO*
}

\author{
INTELLECTUALS AND NEW RIGHT IN ARGENTINA: \\ EXPERTS, MEDIA AND IDEOLOGISTS IN THE PRO CONSTITUTION
}

\author{
DR. ADRIÁN PULLEIRO** \\ Universidad de Buenos Aires/Universidad Nacional de La Pampa \\ Argentina \\ Email: adrianpulleiro@yahoo.com.ar \\ Id-ORCID:0000-0002-4062-4286
}

\begin{abstract}
RESUMEN
En el marco de la crisis argentina de 2001-2002, emergió Propuesta Republicana (PRO), una nueva formación política que en 2007 llegó a la alcaldía de la Ciudad de Buenos Aires y en 2015 conquistó el gobierno central. El objetivo de este artículo es indagar el papel que en el proceso de emergencia y consolidación del PRO jugaron un conjunto de intelectuales que se desempeñaron en tareas de "asesoría" y en ciertos think thanks. Se analizan las acciones y discursos producidos en el seno de experiencias históricas concretas que cruzan prácticas políticas, dinámicas institucionales y trayectorias intelectuales. Priorizamos el período 2007-2015, ya que marca la etapa de consolidación del PRO y permite advertir cambios
\end{abstract}

\begin{abstract}
In the framework of the Argentine crisis of 20012002, PRO emerged, a new political formation that in 2007 reached the City Hall of the City of Buenos Aires and in 2015 it conquered the central government. The objective of this article is to investigate the role played in the process of emergency and consolidation of the PRO by a group of intellectuals who worked in "advisory" tasks and in certain think thanks. We analyze actions and discourses produced within concrete historical experiences that cross political practices, institutional dynamics and intellectual trajectories. We prioritize the 2007-2015 period, since it marks the consolidation phase of the PRO and allows us to notice changes in the role and functioning of
\end{abstract}

* $\quad$ Recibido: 6 de marzo de 2019; Aceptado: 3 de abril de 2019.

** Artículo científico. Este trabajo es parte del proyecto de investigación posdoctoral "Intelectuales en la Argentina del Bicentenario (2008-2012): ideas, formas de asociación y modos de intervención pública. Financiado por CONICET. 
en el papel y el funcionamiento de las prácticas y espacios que nos interesa indagar.

Palabras clave: Intelectuales; cultura; política; nueva derecha the practices and spaces that we are interested in investigating.

Keywords: Intellectuals; Culture; Politics; New Rigth

Cómo citar: Pulleiro, Adrián. (2019). "Intelectuales y nueva derecha en Argentina: Expertos, Mediáticos e Ideólogos en la constitución del PRO". Revista Historia Social y de las Mentalidades, 23(1), 189-210. https://doi.org/10.35588/rhsm.v23i1.3767

\section{INTRODUCCIÓN}

En el marco de la crisis que atravesó a la sociedad argentina a fines del siglo pasado y principios del actual, emergió Propuesta Republicana (PRO), una nueva formación política que en 2007 llegó a la alcaldía de la Ciudad Autónoma de Buenos Aires (CABA) y en 2015 conquistó el gobierno central. La constitución del PRO y su proyección posterior fueron una de las tantas derivaciones de la crisis de representación de los partidos tradicionales y la desestructuración de la cultura política ligada a ellos. El PRO se caracterizó desde un comienzo por presentarse como "un partido de lo nuevo", por pretender eludir las disputas ideológicas que definieron históricamente a la política argentina y por construir una maquinaria, electoral primero y de gestión después, basada en saberes especializados del marketing, los estudios de opinión pública y la administración empresarial.

Este artículo se propone indagar el rol que tuvieron, en el proceso de emergencia y consolidación del PRO, un conjunto de intelectuales que se desempeñaron en ciertos think thanks y llevaron a cabo tareas de asesoría más o menos clásicas. En ese sentido, aborda prácticas intelectuales articuladas constitutivamente con la acción política y que por ende conforman una trama única con determinadas estrategias de intervención pública y mediática. Desde una perspectiva que retoma los aportes de la historia intelectual y de la sociología de la cultura, el objetivo es analizar una serie de trayectorias y discursos producidos en el seno de experiencias históricas concretas a la luz de prácticas políticas, espacios institucionales e itinerarios intelectuales de más largo alcance (Altamirano, Para un programa de historia intelectual 10).

Aunque es un fenómeno con raíces previas, la atención estará puesta en el período 2007-2015, ya que marca la etapa de institucionalización del PRO y permitirá observar con claridad cambios relevantes en el papel y el tipo de funcionamiento que se dieron en las prácticas y espacios intelectuales estudiados. A su vez, el análisis se centrará en el think tank partidario, la Fundación Pensar, $\mathrm{y}$ en dos figuras clave por el peso que tienen en tanto referencias públicas y 
asesores muy cercanos a los principales dirigentes del PRO: Jaime Durán Barba y Alejandro Rozitchner.

\section{UN PARTIDO DE CUADROS (Y DE THINK TANKS)}

Como sostienen Morresi y Vommaro el PRO se caracteriza por la heterogeneidad, tanto de procedencias como de perfiles ideológicos (46). Esa diversidad se ha traducido en la existencia de cinco sectores identificables, que han sido fundamentales para comprender los inicios y también el desarrollo posterior de la organización: la "facción de derecha" (integrada por militantes políticos con antecedentes en partidos tradicionales de esa tradición, como la Unión del Centro Democrático), la "facción de los negocios" (conformada por empresarios), la "facción radical" (provenientes de la Unión Cívica Radical), la "facción peronista" (un importante sector que proviene del Partido Justicialista de la Ciudad de Buenos Aires), y la "facción de las ONG" (básicamente graduados en ciencias sociales que se desempeñaban en centros de estudio, organizaciones no gubernamentales y think tanks más clásicos).

Vale adelantar que, como sostienen dichos autores, esta última facción le ha imprimido buena parte del perfil ideológico que constituye la particularidad del PRO en ese terreno ${ }^{l}$. Este sector está compuesto por profesionales que, salvo excepciones, no tenían experiencia partidaria y que en su mayoría se formó en colegios católicos y estudió carreras de grado en universidades privadas. En cuanto al perfil ideológico, esta facción encarna una posición promedio respecto del conjunto de las facciones que conforman el partido, si se tienen en cuenta las posturas acerca de cuestiones ilustrativas como aborto, relación Estado y mercado, protesta social o concepción de la salud y la educación públicas. $\mathrm{Su}$ "catolicismo social" se evidencia en que se muestran conservadores ante cuestiones culturales y políticas (poco propensos a la legalización del aborto y consideran que los sindicatos tienen un poder excesivo, por ejemplo) y al mismo tiempo son quienes más defienden el rol interventor del Estado en la economía y tienden a no concordar con la idea de que en materia de derechos humanos solo "hay que mirar hacia adelante" (Vommaro y Morresi 51-52).

En los orígenes de la conformación del PRO como partido jugó un papel central la Fundación Creer y Crecer. Constituida en 2001 con Mauricio Macri

1 Vale como indicador de ese peso el hecho de que cuatro de los once miembros del primer gabinete conformado por Macri en el Gobierno de la CABA pertenecían a esta facción: Horacio Rodríguez Larreta (Jefe de Gabinete); Jorge Lemus (Ministro de Salud); Mariano Narodowsky (Educación); y Marcos Peña (Secretario General). 
como presidente y auspiciada por, el también empresario, Francisco de Narváez, esa usina de ideas fue una plataforma pensada para proyectar la carrera política de ambos. Nació con el objetivo de armar equipos técnicos y definir políticas públicas. Aunque duró poco más de un año, ya que la ruptura entre Macri y de Narváez implicó su cierre a fines de 2002, se trató de una experiencia significativa. Allí llegaron a trabajar unas 300 personas. Entre ellos, Marcos Peña-Secretario General del Gobierno de la CABA y luego Jefe de Gabinete de la Nación- se sumó a las filas de la Fundación Creer y Crecer luego de su paso por Poder Ciudadano y CIPPEC, dos ONG que estarían también muy relacionadas con el desarrollo del PRO. Asimismo, pasaron por ese espacio Mariano Narodowski, primer Ministro de Educación de Macri en la ciudad, y Eugenio Burzaco, quien asumiría posteriormente diversos puestos en el área de seguridad.

El otro caso que vale la pena mencionar en lo que podemos llamar la prehistoria del PRO es el del Grupo Sophía. Un think tank creado en 1994 por Horacio Rodríguez Larreta - primer Jefe de Gabinete de Macri en la CABA y Jefe de Gobierno de esa ciudad a partir de 2015-. Desde un comienzo su propósito fue reunir profesionales en diversas áreas que tuvieran vocación de intervenir públicamente. El propio Larreta combinaba un perfil técnico y de gestión, puesto que entre 1995 y 2001 se desempeñó en varios puestos de la administración pública en los gobiernos de Carlos Menem primero y de Fernando de la Rúa más adelante (Rodríguez Larreta fue Gerente general de la Administración Nacional de la Seguridad Social y Director de la Dirección General Impositiva). El Grupo Sophía aportó una cantidad importante de cuadros técnicos que tuvieron su primera experiencia de gestión en el gobierno del PRO en la CABA. Fue el espacio que operó como puente para el arribo a la política de figuras como María Eugenia Vidal - primero ministra de Desarrollo Social de la Ciudad y Gobernadora de la Provincia de Buenos Aires desde 2015-, Carolina Stanley -sucesora de Vidal y luego Ministra de Desarrollo Social del gobierno Nacional-, Sol Acuña -Ministra de Educación de la CABA- y Ramiro Tagliaferro -intendente del municipio bonaerense de Morón.

Si retomamos la idea de que el PRO se conformó a partir de cinco afluentes, podemos concluir que en un momento de crisis de representación como la que caracterizaba a la Argentina a inicios del siglo XXI, tanto los empresarios como los cuadros de perfil técnico que trabajaron en esas usinas de ideas y otras ONGs fueron fundamentales para volver creíble la imagen de un partido que se presentaba como nuevo y "de lo nuevo" (Vommaro y Morresi 38). Los militantes y dirigentes con pasado en los partidos tradicionales (peronismo, radicalismo, liberal-conservadores) que se sumaron a la fuerza encabezada por Mauricio Macri no estaban en condiciones de jugar un papel preponderante en 
ese aspecto y tampoco en lo referido al otro gran pilar de la identidad PRO: la capacidad para llevar a cabo una gestión de gobierno moderna y eficiente.

Desde una perspectiva histórica, cabe agregar dos cuestiones más. Este papel inicial cumplido por las usinas de ideas mencionadas en la conformación del PRO fue discontinuo pero prolongado en el tiempo. La función que el Grupo Sophia y la Fundación Creer y Crecer jugaron en la etapa previa al arribo al gobierno de la CABA tuvo su correlato en la Fundación Pensar durante los años posteriores. A su vez, este es un rasgo que liga al PRO con la concepción de la relación entre saber experto y política que caracterizó históricamente al neoliberalismo en tanto movimiento intelectual. Aunque en la propuesta de los "padres fundadores" había una demarcación más nítida entre especialista y político, basada en la idea de que los expertos debían influenciar a los funcionarios por medio de los líderes de opinión y del convencimiento de la opinión pública, existía en los núcleos originarios del movimiento neoliberal una visión estratégica respecto de la circulación de las ideas. Por citar un caso emblemático, el propio Friedrich Hayek insistía en la necesidad de volcar esfuerzos para editar libros y aparecer en los medios masivos, en el marco de lo que consideraba un verdadero "combate ideológico" (Mato 33). Del mismo modo, Hayek sostenía la necesidad de incidir y formar a periodistas, con especial énfasis en el campo del periodismo económico. En definitiva, si bien más adelante se volverá sobre este punto, es interesante remarcar aquí que para la tradición neoliberal la labor intelectual estuvo desde un principio vinculada con una impronta de intervención pública y con la idea de construir un verdadero ejército de predicadores (Mato 34) ${ }^{2}$.

\subsection{La Fundación Pensar}

La Fundación Pensar (FP) empezó a funcionar formalmente en 2005 y a partir de allí tuvo tres etapas. La primera correspondió al momento de arribo del PRO al gobierno de la CABA y las dos siguientes giraron en torno de las elecciones presidenciales de 2011 y 2015.

En un primer momento, hasta 2009, tuvo un peso importante un núcleo de profesionales con pasado académico que se había acercado al espacio liderado por Macri y que mantenía vínculos personales entre sí. Allí se destacan Humana de 1949, sostenía que los valores son el motor de las conductas y que era imprescindible conocer esos valores cabalmente por medio del trabajo de científicos sociales. En efecto, ese objetivo estuvo en la base de la creación de la Sociedad Mont Pèlerin en 1947 y desde allí de decenas de usinas de pensamiento en todo el mundo (Murillo 37). 
Gabriela Michetti (luego vicejefa de Gobierno y Vicepresidenta de la Nación), el consultor Sergio Berensztein, Gerardo Bongiovanni (fundador y titular de la Fundación Libertad) ${ }^{3}$, Eugenio Burzaco y Jorge Triaca (Ministro de Trabajo de la Nación). De hecho, en esa primera fase este último asumió la Dirección Ejecutiva y Bongiovanni la presidencia.

En esta etapa el think tank del PRO asumía como misión trabajar por "una Argentina próspera, republicana e integrada al mundo" y en su web se autodefinía como "aliada indiscutible de la democracia y el estado de derecho". No se presentaba como una entidad dependiente del partido comandado por Macri, aunque para ese momento su staff estaba plagado de dirigentes y legisladores del PRO.

Desde un comienzo los lazos internacionales fueron una apoyatura para desplegar el proyecto de la FP. La Fundación para el Análisis y los Estudios Sociales (FAES) del Partido Popular de España actuó como un modelo a seguir y como un aliado clave en la organización de eventos y el acceso a financiamiento. De hecho, uno de los primeros eventos de importancia que protagonizó la FP fue el seminario "Nuevas generaciones de líderes de América latina", coorganizado por ambas usinas en marzo de 2008. En suma, FAES fue un puente para que la FP pudiera acceder a financiamientos importantes como los de la Agencia Estadounidense para el Desarrollo Internacional (USAID) y contactos directos con otras fundaciones de la derecha europea (Vommaro et al. 145).

Cuando el PRO accedió al gobierno de la CABA en 2007 la idea original para la FP era mantener un trabajo ligado a las aspiraciones presidenciales de Macri mediante la elaboración de propuestas para el ámbito nacional. Sin embargo, el hecho de que el PRO se convirtiera en una fuerza de gobierno en la ciudad fue de suma importancia para la FP ya que terminó siendo una referencia ligada a la gestión, lo cual generó condiciones para probar ideas y ser vista como una oportunidad de empleo, sobre todo, para intelectuales jóvenes. Este perfil, que con los años se fue explotando mucho más, colocó en un lugar relevante a la posibilidad de sumar jóvenes a los equipos de gobierno y a las filas partidarias (Echt 103). En este caso con una afluencia predominante de graduados de la Universidad Católica Argentina, la Universidad Torcuato Di Tella y la Universidad San Andrés. 1988 "trabaja en la investigación y difusión de temas de políticas públicas", "promoviendo las ideas de la libertad, el republicanismo, la democracia y el Estado de Derecho". Creada por un grupo de empresarios, profesionales e intelectuales, cuenta con el apoyo de más de 200 empresas privadas. Integra la Fundación Internacional para la Libertad, que preside Mario Vargas Llosa y la Red Liberal de América Latina (www.libertad.org.ar). 
En 2009, Jorge Triaca fue electo Diputado Nacional y abandonó el cargo de Director Ejecutivo. Esto terminó de formalizar un nuevo momento en la organización, que estuvo guiado por la aspiración presidencial de Macri para las elecciones de $2011^{5}$. Francisco Cabrera fue el hombre clave en esa reorganización. Procedente del mundo empresarial y parte del círculo íntimo de Macri, Cabrera mantuvo su cargo en el gobierno de la CABA, como ministro de Desarrollo Económico, y asumió como presidente de la FP. Desde ese lugar actuó como nexo entre el trabajo de la FP y el PRO. De ese modo, la fundación pasó a tener un rango mayor entre las prioridades de esa fuerza política y una labor más cercana al propio Macri.

Cabrera se encargó de reforzar el perfil intelectual de la fundación convocando a Miguel Braun para desempeñarse como Director Ejecutivo y a Iván Petrella para asumir como Director Académico. Braun es uno de los fundadores del Centro de Implementación de Políticas Públicas para la Equidad y el Crecimiento (CIPPEC), donde fue director entre 2000 y 2010. Se acercó poco antes al PRO a través de Rodríguez Larreta. Además de pertenecer a una importante familia empresaria local ${ }^{6}$ tenía las acreditaciones académicas suficientes para relanzar la FP: economista de la Universidad de San Andrés, y Doctor y Magíster en Economía de la Universidad de Harvard. Especialista en temas fiscales, con experiencia docente en las universidades de Buenos Aires, San Andrés y Torcuato Di Tella, también había trabajado como consultor del Banco Interamericano de Desarrollo y el Banco Mundial. Por su parte, Petrella estaba recién vuelto al país luego de vivir más de diez años en Estados Unidos, donde desarrolló el grueso de su carrera académica. Se recibió de Licenciado en Relaciones Internacionales en Georgetown y. al igual que Braun, obtuvo su doctorado en Teología en Harvard. Más allá de ser hijo del ex vicecanciller Fernando Petrella (1992-1996), al momento de ingresar a la FP no tenía experiencia política y encabezaba una cátedra en la Universidad de Miami. En su caso la vía de contacto con el PRO fue Marcos Peña, un amigo de la adolescencia. Una vez instalado en el país también se dedicó a la docencia en la Universidad Torcuato Di Tella y en la de San Andrés. Tiempo después tanto Petrella como Braun asumirían cargos políticos. El primero fue elegido Legislador en la CABA en 2013 y fue funcionario del Ministerio de Cultura de la Nación entre 2015

$5 \quad$ Ver "El gabinete en las sombras que trabaja para Macri 2011", La Política Online, 2/4/2010.

6 La familia Braun es una tradicional familia de la Patagonia que basó su fortuna en la actividad comercial. Federico Braun, tío de Miguel Braun y de Marcos Peña Braun, comanda el grupo económico que es dueño de la cadena de supermercados La Anónima. La cuarta cadena del país por volumen de ventas. 
y 2017. Braun fue Secretario de Comercio de la Nación y luego Secretario de Política Económica del Ministerio de Hacienda con la llegada de Macri a la presidencia.

Durante esta etapa, la FP reunió en su consejo de Administración a muchos de los dirigentes más importantes del PRO. Entre ellos a Marcos Peña, Horacio Rodríguez Larreta, Esteban Bullrich, María Eugenia Vidal, Sergio Bergman, Jorge Triaca y Federico Pinedo. También tuvieron su lugar en esa instancia el consultor Luis Secco y el filósofo Alejandro Rozitchner, junto con Gerardo Bongiovanni, quien siguió participando en paralelo a su condición de presidente de la Fundación Libertad.

En el nuevo esquema la FP dejó progresivamente atrás la centralidad de los eventos, -la organización de conferencias y seminarios fundamentalmente había marcado el primer período-y pasó a colocar el foco en la generación de conocimiento con fines prácticos. El método de funcionamiento se orientó hacia ese propósito y se basó en dos líneas prioritarias (Echt 74-75). Por un lado, mesas de trabajo para debatir temas estratégicos y políticas públicas a nivel nacional. Cada mesa estaba coordinada por un facilitador con trayectoria en el sector (según el caso ex funcionarios, intelectuales o empresarios). Macri se reunía periódicamente con las distintas mesas de trabajo para conocer los avances en los planes de gobierno para las distintas áreas. Un segundo eje fue la producción sobre temas coyunturales, ya sea como insumo para las intervenciones públicas de las principales figuras del partido como para debates parlamentarios. A su vez, la labor de la FP tuvo consecuencias en otros dos planos fundamentales para la construcción política. La apertura de instancias para la discusión en torno a las propuestas elaboradas siguió siendo una herramienta relevante para la construcción de redes a nivel local e internacional ${ }^{7}$. Al tiempo que la FP se convertía en un espacio importante para reclutar nuevos militantes y cohesionar a los dirigentes intermedios de un partido en plena expansión. De algún modo, la fundación operó como una escuela de cuadros en el sentido clásico, generando condiciones para la transmisión de ideas y valores y consolidar una forma de entender y llevar a adelante la práctica política ${ }^{8}$.

Cuando Macri desistió de competir por la presidencia en 2011 y volcarse a obtener la reelección como Jefe de Gobierno de la CABA el proyecto de la

7 Más allá del nuevo énfasis planteado, la FP no abandonó la organización de eventos. De hecho, en octubre de 2010 se realizó la segunda edición del "Campus FAES", un seminario organizado por FAES que contó con Macri como anfitrión y que fue patrocinado por la FP y la Fundación Libertad de Rosario.

8 Ver "El macrismo puso en marcha un semillero de dirigentes", La Nación, 24/11/2014. 
FP entró en crisis. Sin embargo, poco después la dirección del PRO asumiría el horizonte de las presidenciales 2015 y en ese marco la FP fue objeto de nuevos esfuerzos para darle más visibilidad como usina de ideas y avanzar en una mayor profesionalización ${ }^{9}$.

Una de las medidas más significativas fue contratar a un especialista en comunicación que se encargó de diseñar y dirigir un plan con dos objetivos fundamentales: ganar más legitimidad en el partido como espacio de producción de conocimiento especializado y sumar presencia en el espacio público a través de voceros que tuvieran más lugar en los medios masivos (Echt 78). En suma, se trataba de asumir una acción más agresiva con un destinatario claro: si el equipo de comunicación partidario se dirigía al votante, la comunicación de la FP se dirigía a los formadores de opinión.

Ampliar las temáticas abordadas para ir más allá que la economía fue otra línea de acción. No solo por una necesidad política, sino también de imagen ya que estaba en juego la necesidad de "humanizar" a la fundación y con ello al imaginario que rodeaba al PRO. El trabajo se organizó en tres grandes bloques: económico, social e institucional. Cada bloque estaba integrado por varias áreas (30 en total) con sus respectivos proyectos y estaba a cargo de un coordinador ${ }^{10}$. En todos los casos se involucraron los principales referentes del partido.

La otra función clave de la FP en esta nueva etapa estuvo dada por la generación de contenidos discursivos para las campañas electorales. En este caso en coordinación con el equipo de comunicación del PRO, que solicitaba sobre todo datos que luego se encargaba de adaptar a distintos formatos. De este modo, a partir de 2011 la FP vivió un proceso de institucionalización y expansión significativo. En un plano, se multiplicaron las sedes provinciales (para 2016 había 15 funcionando). Para 2015 contaba con unas treinta personas trabajando cotidianamente, la mayoría con contratos, un amplio número de consultores contratados ad hoc para estudios específicos, y unos trescientos involucrados en la modalidad de voluntariado, incluyendo a los funcionarios y dirigentes partidarios (Echt 102). No obstante, luego del triunfo electoral y el arribo al gobierno nacional en 2015 la sede central de la FP quedó virtualmente paralizada. A principios de

$9 \quad$ Ver “La Fundación Pensar se prepara para 2015”, El estadista, 7/5/2014.

10 Una de las producciones más representativas del período fue la propuesta de política agroindustrial que fue presentada en diversos eventos públicos y elaborada por un equipo que cruzó a especialistas con miembros de entidades empresariales del sector. Decimos representativa porque ilustra el modo de trabajo y contiene buena parte de los principios que el gobierno de Macri puso en práctica en materia económica (liberalización y apertura). Así aparece en "La usina del eterno retorno", Revista Crisis, 6/11/2015. 
2018 el PRO tomó la definición de relanzarla de cara a la carrera por la reelección, aunque sin el equipo que estuvo al frente desde 2010.

Tal como se ha señalado a lo largo de este apartado, podemos sostener que la FP fue un factor relevante en el proceso de consolidación del PRO y su proyecto político. Su rol más evidente estuvo dado por el aspecto ideológico y por constituirse en un espacio de sistematización y elaboración de propuestas y discurso político, esto más allá de que excede los límites de este trabajo una indagación cuantitativa sobre su producción y una evaluación de cuánto esa producción estuvo presente en las políticas públicas aplicadas. Junto con eso, además de ofrecer instancias para la formación de cuadros técnicos. la FP actúo como un dispositivo de reclutamiento y cohesión identitaria. Y en un tercer nivel fue una herramienta para respaldar la idea de que el PRO era una experiencia política nueva y diferente a las demás, sobre todo por lo que permitió transmitir con su propio funcionamiento, al mostrar que el PRO apostaba por la planificación y desestimaba la improvisación. Del mismo modo, la impronta ligada al conocimiento y al diagnóstico metódico encarnado en un plantel de expertos reforzó el carácter post ideológico con el que el PRO se asoció en toda la trayectoria que lo llevó al gobierno nacional. Contar con un plantel de especialistas refuerza la idea de que es posible, mediante un conocimiento técnico, elevarse por sobre otras posturas acerca de temas estratégicos que se presentan como dogmáticas. Finalmente, la FP aportó elementos para proyectar la imagen de una fuerza política pluralista, capaz de dialogar e incorporar ideas diversas. La metodología de elaboración de propuestas en las que se buscó involucrar a expertos y dirigentes sectoriales no partidarios tuvo esa pretensión. Y ligado a esto, la idea de que en la fundación se estaban formando los equipos de gobierno para el futuro también abonó a ese pilar del discurso macrista que destaca al trabajo en equipo frente al personalismo que se achaca en otras fuerzas políticas.

\section{DURÁN BARBA: MUCHO MÁS QUE UN CONSEJERO EN LAS SOMBRAS}

Como plantean los autores de Mundo PRO, Jaime Durán Barba se reveló con los años como una figura central en el armado de las estrategias del PRO (164). No obstante, su papel va más allá de la mera asesoría en cuestiones de comunicación política. Su peso es tan importante que en sus orientaciones el PRO encontró en gran medida el sostén de sus rasgos ideológicos vinculados con la reivindicación del pragmatismo y el rechazo de las grandes tradiciones culturales e identidades políticas que le dieron forma a las disputas sobre el rumbo del país durante gran parte del siglo XX. Indicar que el papel de Durán 
Barba va más allá de la figura de un técnico y que actúa como un asesor-ideólogo que extendió su influencia al conjunto de la dirigencia del PRO es la hipótesis con la que trabajamos este apartado.

Los datos centrales de la trayectoria del asesor ecuatoriano son conocidos (Giordano, Natanson), pero destacamos algunos que son relevantes para nuestro objetivo. Durán Barba es consultor de imagen y experto en estudios de opinión pública. En 1980 fundó Informe Confidencial Ecuador, una empresa dedicada a los sondeos desde donde fue construyendo una carrera que se expandió regionalmente. Desde hace más de dos décadas colabora en la Graduate School of Political Management de la George Washington University.

Cuando era un estudiante de filosofía en los años ' 70 hizo una estancia en Argentina, en la Universidad de Cuyo, atraído por las figuras de los filósofos Enrique Dussell y Arturo Roig. Luego del golpe de Estado de 1976, se trasladó a la Patagonia para estudiar en la Fundación Bariloche, donde se formó en las técnicas y la metodología de la sociología empírica clásica.

Antes de convertirse en una pieza clave para la consolidación de la referencia pública de Mauricio Macri y de su fuerza política, Durán Barba fue funcionario del Gobierno de Jamil Mahuad, durante los dos años que duró su mandato, entre 1998 y 2000. Desde su puesto en la Secretaría de la Administración Pública cumplió un papel central en las conversaciones de paz con Perú y en el diseño de la política económica que terminó en la dolarización. La relación entre Durán Barba y Macri data de 2003. Se conocieron por medio del entonces operador macrista Juan Pablo Schiavi, antiguo cuadro de SOCMA (empresa familiar de los Macri) con vínculos estrechos con el ex presidente Carlos Menem, por entonces en plena pelea por la presidencia. A partir de 2005, cuando Macri se impuso en las elecciones a Diputados Nacionales, Durán Barba se constituyó en el pilar de las campañas electorales de su cliente argentino. De ahí en más su opinión ha sido clave en la estrategia asumida por el ahora presidente y su partido. Sin ir más lejos, los trabajos citados coinciden en atribuirle al ecuatoriano un papel central en la decisión de modificar la idea inicial de disputar la presidencia en 2011 y de rechazar una posible alianza con un sector del peronismo en 2015. Llegados a este punto nos interesa indagar en el sistema de ideas y creencias que proyecta Durán Barba en su actividad intelectual, en tanto asesor-ideólogo. Para ello nos vamos a nutrir de dos de sus libros que más repercusión tuvieron. Mujer, sexualidad, internet y política. Los nuevos electores latinoamericanos (Fondo de Cultura Económica, México, 2006). Y El arte de ganar: cómo usar el ataque en campañas electorales exitosas (Debate, Buenos Aires, 2010), ambos escritos con su socio Santiago Nieto. 
Los libros de Durán Barba son presentados como el resultado de su experiencia como consultor político. El testimonio se propone como una fuente de legitimidad y su expertise aparece vinculada a una base empírica, opuesta a un tipo de conocimiento cuestionado por abstracto y enciclopédico. De ese modo, estos materiales refuerzan una posición de enunciación que tributa en una figura de intelectual ligada a un saber aplicado, que remite simultáneamente a un método científico de trabajo. Como veremos, se trata de una figura ajena al pensamiento crítico; es decir, al ejercicio de distanciamiento, de historización y de indagación de las múltiples determinaciones que están en la base de cualquier realidad social y que conforman las dimensiones de ese tipo de elaboración intelectual.

En Mujer, sexualidad, internet y política hay una tesis ordenadora: las elecciones se ganan con el voto de las mayorías. Por ende, para los autores, las características de esas "nuevas masas" latinoamericanas deben ponerse en el centro de las preocupaciones de políticos y especialistas. Los títulos de los capítulos del libro ilustran los intereses que los guían y la perspectiva de análisis que asumen: "El nuevo elector latinoamericano"; "La agonía de las grandes utopías: el mundo en el que nace el nuevo elector"; y, por último, "Por qué votan los electores".En ese esquema Durán Barba y Nieto vuelven sobre una tesis poco original. Sostienen que la crisis de las ideologías modernas repercute en un trastocamiento en el papel tradicional de los intelectuales: "la mayoría de los electores contemporáneos se interesan poco en sus discusiones y se mueven en el escenario electoral tratando de satisfacer necesidades concretas, o satisfaciendo sentimientos de envidia, solidaridad, confianza, respondiendo a identidades grupales regionales o étnicas o simplemente porque les gusta un espectáculo" (Durán y Nieto, Mujer, sexualidad, internet y política 46). Nos interesa adelantar que aquí lo que puede ser parte de un diagnóstico válido se constituye en sustento de una valoración más bien cuestionable cuando los autores hablan de "una población que maneja sus destinos a su manera" (Durán y Nieto, Mujer, sexualidad, internet y política 71). De esa forma, los procesos políticos se plantean como una variable -o como un efecto- del comportamiento de esa "gente común".

Vale decir también que, si bien los títulos de estos libros y sus temas centrales están ligados a un campo de saberes específicos, sus destinatarios no son otros especialistas. Esos títulos y temas parecen ser excusas para desplegar una abnegada y sistemática labor de divulgación. Es en ese punto en donde la acción de estos especialistas entronca con una de las facetas que hace a la manera de entender el rol de los intelectuales en la tradición neoliberal, en la cual -como hemos señalado más arriba- éstos han sido históricamente concebidos 
como auténticos predicadores. Lo que paradójicamente, hace que ese accionar divulgador de Durán Barba remita a la tradición del intelectual moderno ${ }^{11}$. A su vez, hay que decir que son libros que entran en la categoría best-seller, algo sobre lo que volveremos más adelante.

En El arte de ganar, las ideas centrales refuerzan los planteos medulares del libro anterior, aunque en este caso se pone más dedicación en todo lo que rodea a las campañas electorales. A eso se suma una reivindicación más directa del rol de los autores como consultores políticos. Así las cosas, en el punto de partida hay un diagnóstico incuestionable, pero que fiel al estilo duranbarbiano ese diagnóstico se toma como algo dado y no forma parte de los hechos a problematizar en el desarrollo del propio análisis: las estrategias electorales se han modernizado y las campañas se han profesionalizado al punto de volver imprescindibles las tareas de los especialistas. Para superar la "vieja forma de hacer política", Durán Barba y su socio sostienen que hay que apoyarse en profesionales capaces de diseñar un plan en el que nada quede librado a la improvisación y respecto del cual los distintos actores participantes (candidatos, dirigentes partidarios, equipos de comunicación) deben sostener un apego estricto.

Por otra parte, la orientación es clara: pasar de "la agenda para las élites" a la de los "electores comunes". Es decir, poner en el centro de la estrategia a las herramientas de comunicación masivas para llegar sobre todo al votante despolitizado y más desinformado. Lo que lleva a su vez a apuntar más a las audiencias y las redes sociales que a la militancia cara a cara y a los formatos más tradicionales. Finalmente, si el interés de los votantes no pasa por grandes gestas, sino por los problemas de todos los días, el destinatario de la estrategia comunicacional es esa persona común a quién hay que resolverle los problemas. El discurso debe contener a ese vecino de a pié como principal protagonista. Como consecuencia la política "cercana a la gente" no pretende ser una herramienta de transformación colectiva sino un medio para la gestión de los problemas. $\mathrm{He}$ aquí un rasgo central en el ideario PRO.

Hay aquí, entonces, una serie de núcleos significantes que emergen de la práctica intelectual de Durán Barba como un sistema de ideas que estructura esa práctica: hay una sociedad conformada por individuos racionales que son capaces de elegir a sus representantes de acuerdo a quienes se acercan más a lo que cada

11 Como sostiene Carlos Altamirano los intelectuales son una "especie moderna". "El intelectual no es sólo el hombre que piensa el mundo sino el que trasmite a otros hombres lo que piensa del mundo" (Altamirano 101). Asimismo, "todas las grandes narrativas de la modernidad, sea el progreso, la de la nación o la del pueblo, así como el conjunto de los 'relatos militantes' de los siglos XIX y XX, proceden de las filas de la intelligentsia" (Altamirano 104). 
quien necesita ${ }^{12}$. Las contradicciones de esta concepción sobre la sociedad, sobre los comportamientos humanos y los valores que priman en un momento dado son tan conocidas como las críticas históricas al pensamiento liberal y a las corrientes de la sociología funcionalista. Solo para mencionar las principales: la división simplificadora entre individuo-sociedad; la incapacidad para incorporar las relaciones de poder y la desigualdad simbólica, informativa, cultural; el revés que se plantea acerca de la relación clásica entre (des)información y autonomía. No obstante, nos interesa remarcar dos cuestiones. La primera es que, como ya adelantamos, en el discurso de Durán Barba hay un diagnóstico que se postula como un hecho dado, sobre el cual no hay por qué reflexionar en términos de procesos sociales, y por ende es un diagnóstico que aparece como una situación deseable: individuos cada vez más desinteresados por la cuestiones públicas, que han quedado "liberados" de las grandes tradiciones políticas de antaño, definen los procesos políticos de acuerdo con sus inquietudes más concretas y elementales. Estamos ante un discurso (ilusorio) que refuerza todo un sentido común acerca de la sociedad, los sujetos y sus instituciones, que implica puntualmente el refuerzo de la ideología ${ }^{13}$ neoliberal en uno de sus tópicos más básicos: la concepción de un individuo racional autónomo que además es el máximo responsable de su devenir. Por si hace falta aclarar, la ideología neoliberal no lo cubre todo -en tanto espacio ideológico es terreno de luchas y disputas-, pero su eficacia reside en la configuración de un efecto de totalidad social plena. Efecto al que desde nuestro punto de vista un pensamiento como el de Durán Barca contribuye tanto a partir de sus postulados como de sus procedimientos.

12 Aquí aparece un correlato específico con el modo en que la vertiente neoliberal austronorteamericana concibe el rol social del mercado que nos parece vital, ya que a nuestro entender opera como una matriz de interpretación: "si el hombre sabe comportarse, no es gracias a la "naturaleza", sino gracias al mercado, que constituye un proceso de formación (...) Lo que asegura la integración de las acciones individuales en el conjunto del sistema social de producción es la persecución, por parte de cada individuo, de sus propios objetivos (...) La economía de mercado tiene como condición la libertad individual más completa. Éste es un argumento más funcional que ético: la condición del funcionamiento del mecanismo de mercado es la libre decisión en función de las informaciones que cada uno posee (Laval y Dardot 131)

13 Vale señalar, por un lado, que todo pensamiento interviene en una coyuntura reforzando las formas impuestas de desigualdad y dominación, o pensando contra esas formas, al estilo de una contraideología (Thompson 102-103). A su vez, usamos un término como el de ideología a sabiendas de que es un concepto polémico. Lo hacemos para sostener que en una sociedad basada en la explotación y en el acceso desigual a los recursos materiales y simbólicos, hablar de las prácticas de pensamiento es referirse a un espacio ideológico que se caracteriza por la complejidad, por las luchas que se despliegan en el marco de una tendencia a la unificación de lo heterogéneo. En suma, "se trata de pensar a la ideología como una tendencia dominante a la unificación, administración o disciplinamiento de la heterogeneidad de prácticas de pensamiento. La ideología dominante es, en este sentido 'única', pero no es total" (Romé 8). 
La segunda cuestión para remarcar es que se trata de un pensamiento que, desde el punto de vista formal y de los mecanismos de su circulación, adopta las formas de la industria cultural y más específicamente de la publicidad (slogans, aforismos, afirmaciones de alto impacto). El hecho mismo de que se trate de libros ideados como, y convertidos en, best sellers nos dice mucho de esos mecanismos ${ }^{14}$. En un primer nivel, hablar de un texto que se caracteriza por irrumpir en un momento dado como un éxito de ventas (los de Durán Barba lo fueron) puede decir cosas acerca de los públicos, de la recepción de ciertas ideas en una coyuntura determinada y de la circulación de ciertos significados y valores. En el caso de Durán Barba además nos da la posibilidad de ratificar que su figura, su alcance y el modo en el que su labor intelectual se ha insertado en las disputas político-ideológicas de la Argentina reciente dista mucho de acotarse a su papel de "consejero del Príncipe" o del experto que asesora a una fuerza política. No sólo ha sido clave en las campañas electorales del PRO, como vimos la producción intelectual de Durán Barba también aportó elementos determinantes que hacen a los fundamentos ideológicos de la práctica política de esa fuerza, al tiempo que se constituyó en una voz pública de un ideario neoliberal que la excede.

\section{ALEJANDRO ROZITCHNER Y LA FILOSOFÍA DEL ENTUSIASMO}

El caso de Alejandro Rozitchner puede ser presentado a priori como un polo opuesto a la figura de Durán Barba. De un lado el especialista, del otro el filósofo. Sin embargo, así como hemos descrito la complejidad que asume la labor de Durán Barba, tanto el ideario como el itinerario intelectual de Rozitchner tienen muchos vasos comunicantes con los del ecuatoriano ${ }^{15}$. El papel que desempeña Rozitchner en el mundo PRO nos interesa particularmente porque es

14 En el marco de los procesos de concentración y transnacionalización de la industria cultural que se han desplegado en las últimas dos décadas, el sector editorial ha creado una ingeniería específica para la producción de best-sellers políticos. El éxito de este tipo de libros está basado en una planificación en la que participan editores, agentes, publicistas y demás trabajadores de la industria editorial orientados fundamentalmente por las reglas mercantiles de la oferta y la demanda (Saferstein 201).

15 De hecho, Rozitchner escribió el prólogo de Mujer, Sexualidad, Internet y Política. En ese texto halaga la capacidad de los autores para percibir los cambios que marcan la época y los define como "facilitadores del diálogo" y "terapeutas sociales". En otro texto, Rozitchner asegura haber aprendido de Durán Barba "una visión de la política ligada a los temas humanos, superadora del viejo juego estéril de un poder que nunca termina de entenderse para qué se lo busca" (Rozitchner, Estamos 34). 
sabida su cercanía con Mauricio Macri -tiene una oficina en la Casa de Gobierno y participa cotidianamente en reuniones de gabinete- y por lo que lo diferencia de sus colegas intelectuales en ese mundo, en pocas palabras una formación no anclada en ninguna disciplina con perfil técnico o profesional.

Como Licenciado en Filosofía por la Universidad Central de Venezuela ${ }^{16}$ dictó clases en la Universidad de Buenos Aires desde la transición democrática en los años ' 80 . En ese momento se integró a la cátedra de Filosofía de Tomás Abraham, allí formó parte de un circuito de socialización intelectual que por aquella época significó el contacto con autores poco transitados en la academia, en donde el pensamiento de Michel Foucault ocupaba un lugar vital (Viotti). Desde la década siguiente se desempeñó en los medios masivos. Fue guionista en programas televisivos de humor y fue columnista en programas de radio de corte juvenil.

En 1993 publicó, junto con el conductor Mario Pergolini, un libro titulado Saquen una hoja. Si bien venía de publicar otros títulos: un libro de filosofía para niños, uno sobre la cultura del rock y un tercero sobre el músico Luis Alberto Spinetta, con ese texto inauguraría toda una saga de manuales morales a mitad de camino entre los libros de autoayuda y los ensayos filosóficos. En aquel texto dedicado a la escuela secundaria insistía en la necesidad de fomentar la creatividad frente a una educación catalogada como burocrática y aplanadora del entusiasmo.

La crisis de 2001 representó un quiebre para Rozitchner. La política, que hasta ahí había sido un tema secundario en su producción, pasó a ocupar un lugar central en su práctica intelectual. Publicó varios libros en los que empezó a desplegar su filosofía del entusiasmo para interpretar las salidas deseables a la crisis ${ }^{17}$ y empezó a participar del programa televisivo Hora Clave, un clásico del género conducido por el abogado y periodista Mariano Grondona.

Unos años después se acercó al PRO, donde encontró una afinidad con lo que venía pregonando en los cursos dictados sobre filosofía del entusiasmo en espacios privados y empresas. Tejió una relación de confianza con Macri y una relación personal con Marcos Peña. Se sumó a la Fundación Pensar, en donde

16 En los '70 se exilió en ese país con su familia. Su padre, León Rozitchner, fue una figura relevante en la cultura de izquierdas argentina.

17 De la producción de crisis, a la producción de país (Libro del Zorzal, 2002); Ideas falsas. Moral para gente que quiere vivir (Del nuevo extremo, 2004); Amor y País. Manual de discusiones que perdí (Sudamericana, 2005); Pensar para hacer. Cómo transformar la filosofía en una experiencia real (Santillana, 2006); y Ganas de vivir. La filosofía del entusiasmo (Penguin Random House, 2012). 
ofreció sus cursos filosóficos. Con el tiempo se consolidó como asesor en la mesa chica del PRO.

Dicho esto, es importante poner el foco en una figura intelectual que también cumplió un doble papel. Analizar las intervenciones públicas y los textos de Rozitchner permite asomarse a otro de los pilares ideológicos que componen el ideario PRO. Paralelamente, hacer esa indagación supone dar cuenta de una de las vías con las cuales esa formación política participa en las disputas culturales de su tiempo.

Tomadas en conjunto esas producciones ofrecen una serie -en el sentido fuerte del término-, de lecciones morales que adaptan a la filosofía al formato de la cultura de masas. Lenguaje simple, ejemplos efectistas, razonamientos sencillos. Sus libros, ideados como best sellers aunque no lleguen a ser un gran éxito de ventas y publicados por los tanques de la edición o por pequeñas editoriales, vehículizan el individualismo contemporáneo; están dedicados a la autonomía, el bienestar personal y familiar, la religión, la educación, el rock y, más recientemente, la política (Viotti). En esa serie nos interesa destacar el modo en que Rozitchner presenta su filosofía del entusiasmo y qué le permite decir esa filosofía sobre la política y la sociedad argentinas.

En principio, como ocurre más implícitamente con el método de Durán Barba, su filosofía es una filosofía anti-crítica; es parte de una cruzada contra el pensamiento crítico. En su Ganas de vivir. La filosofia del entusiasmo (Sudamericana, 2012) "la crítica" es sinónimo de un "estadio adolescente" que deriva en una "actitud esterilizante". Esta imagen, que puede ser proyectada al nivel individual y colectivo, sin ninguna contradicción, está en la base de su diagnóstico y su propuesta para el futuro del país.

En su último ensayo, La evolución de la Argentina (Mardulce, 2016), Rozitchner propone al entusiasmo, la creatividad y el deseo como motores de un cambio político encarnado sin más en la alianza gobernante Cambiemos. En su opinión, hay una fuerza que se opone a esa evolución y es "el conflicto".

El planteo no es original pero sí arriesgado. Para fundamentarlo, Rozitchner recurre a la idea de la responsabilidad individual, tan cara al pensamiento neoliberal, y a la negación de la historia, un mecanismo básico de toda ideología dominante. De este modo, niega la idea de que existan derechos dados de antemano. Y con ello postula una ruptura con la concepción misma de los derechos humanos y sociales. En su lugar propone una "moral de acción" centrada en el "aquí y ahora": los derechos hay que ganárselos. Ya no se trata de demandar derechos sino de (auto)garantizárselos. La figura de la autorrealización y del emprendedadurismo son la cristalización lógica de esa concepción. La consecuencia política también es clara: las trayectorias sociales dependen de cada quien y no de los marcos institucionales, de las normas y 
de las condiciones históricas derivadas de las luchas en las que los colectivos humanos están insertos.

A su vez, Rozitchner rechaza la idea de que el proyecto de Cambiemos suponga redefinir un relato histórico, como cualquier otro proyecto político con aspiraciones hegemónicas. Es más, la historia parece ser un terreno que no hay que pisar, al punto de sostener que "la historia es tiempo muerto, está llena de personas muertas" y "no hay libertad en el pasado".

En este sentido, la filosofía de Rozitchner ofrece una actualización de los preceptos neoliberales ligados a la felicidad individual y a la concepción liberal clásica que aspira a una sociedad sin conflictos. En sus palabras, el ideario del PRO, se puede sintetizar en una serie de ideas:

“...El respeto a la ley (que en la Argentina es ya todo un programa revolucionario), la necesidad de lograr instituciones fuertes e inteligentes, la política como servicio al ciudadano (...) Creemos en el proyecto personal, creemos que detrás del abusivo y equívoco concepto de 'pueblo' hay personas que quieren vivir y a las que es bueno ayudar a vivir. Creemos en la necesidad de generar condiciones para que esa diferencia florezca, porque la sabemos productiva. Queremos desarrollo, no lucha, queremos crecimiento, obras, cercanía (...) Tal vez esa diferencia entre una política de lucha y una política de desarrollo lo resuma todo" (Rozitchner, Estamos 35).

Se trata, en suma, de un discurso que encuentra su eficacia en los sentidos comunes que refuerza (el éxito es un logro personal y depende del esfuerzo individual; los conflictos sociales son un obstáculo para el progreso; el ejercicio de la crítica es algo aburrido que lleva a la parálisis; la atención sobre el pasado no permite una visión superadora de cara al futuro), pero también en lo que aporta como complemento: una nueva utopía individualista ${ }^{18}$.

La filosofía del entusiasmo que predica Rozitchner tiene muchos puntos de contacto con las proclamas sobre la importancia del "factor humano" que abundan en la en la literatura del neomanagement. Si en la sociedad industrial, el sujeto a construir era el sujeto productivo, en la actualidad se trata de un "sujeto empresarial" o "neosujeto"; "Se trata de gobernar a un ser cuya subjetividad debe estar implicada en la actividad que se requiere que lleve a cabo (...) El sujeto unitario es, por lo tanto, el sujeto de la implicación total de sí. El blanco del nuevo poder es la voluntad de realizarse uno mismo en el proyecto que se quiere llevar a cabo (...) Porque el efecto buscado por las nuevas prácticas de fabricación y de gestión del nuevo sujeto es hacer que el individuo trabaje para la empresa como si lo hiciera para él mismo, suprimiendo así todo sentimiento de alienación (Laval y Dardot 331-332). 
De modo tal, la filosofía del entusiasmo de Rozitchner propone una utopía y a su vez remite a una forma de pensar que produce un efecto de cercanía que es coherente con el perfil ideológico del PRO. Es decir, a un modo de ejercer la reflexión que propone explícita e implícitamente evitar la complejidad y que proyecta un modelo específico de quehacer intelectual. La eficacia de la filosofía de Rozitchner está ligada a una actitud anti aristocrática, ya que si generar ideas es algo "fácil" hay en juego un efecto desacralizador sobre la actividad intelectual. Sin embargo, una mirada más atenta sobre el producto final de esa praxis intelectual nos coloca ante los límites de ese pensamiento. El estilo Rozitchner tiene mucho de lo que Bourdieu llamó un "pensamiento de ideas preconcebidas". El tipo de reflexión desplegada por los llamados pensadores rápidos o mediáticos: “piensan mediante 'ideas preconcebidas', es decir, mediante 'tópicos' (...) son ideas que todo el mundo ha recibido, porque flotan en el ambiente, banales, convencionales" (Bourdieu 39). Lo que nos lleva a un problema más relevante. Este pensamiento de la superficie de las cosas elude, siguiendo a Bourdieu, el problema de la comunicación o de la distancia entre los códigos de producción y los de recepción. Cuando lo que predomina son esas ideas preconcebidas la comunicación es instantánea, como lo que ocurre en las conversaciones cotidianas altamente codificadas. Cuando el sentido es instantáneo se corre de escena lo que el pensamiento encierra como proceso productivo y como posibilidad abierta, o sea como subversión: aquello que depende de desbaratar ideas previas y de demostrar razonamientos nuevos. En otros términos, el caso de Rozitchner niega la posibilidad de lo que anteriormente denominamos pensar contra la ideología dominante, o sea contra los sentidos establecidos que refuerzan lo dado. Y lo hace sin esconder nada. Su filosofía mistifica el propio proceso de producción de las ideas, promueve como ideal un lenguaje evidente -transparente-, que en palabras de Michel Pecheux (2016) postula la literalización como mecanismo comunicativo y por ende el empobrecimiento de la experiencia misma de estar en el mundo.

\section{CONCLUSIONES}

En el proceso de emergencia y consolidación del PRO una capa de intelectuales procedentes de universidades privadas y con formación en las ciencias sociales desempeñó un papel importante. Ese papel puede notarse en el perfil ideológico que asumió esa formación de la nueva derecha argentina y en una serie de iniciativas específicamente intelectuales - fundamentalmente una serie de think tanks - que en distintos momentos oficiaron de usinas de saberes especializados y espacios para la construcción de redes locales e internacionales, la formación y captación de cuadros y la cohesión identitaria. 
Al mismo tiempo, los rasgos que asumió la experiencia del PRO son impensables sin la labor de ciertos intelectuales reconocidos y reconocibles. A lo largo de este texto pudimos ver como la figura de Jaime Durán Barba encarna la autoridad que le confiere su condición de experto y simultáneamente es fundamental para comprender no sólo al PRO como maquinaria electoral y de gestión, sino también para entender el modo en que ese partido irrumpió de la mano de una serie de ideas acerca de la sociedad y la política, cuya consolidación como fuerza de gobierno y su éxito electoral contribuyeron a reforzar. Asimismo, indagar en la figura de Alejandro Rozitchner nos permitió analizar una de las vías desde las cuales el PRO construyó un ideario que retoma tópicos clásicos del liberalismo y los combina con elementos propios de una ideología neoliberal que en las últimas décadas se ha apoyado cada vez más en valores inmateriales y en la apuesta por un mundo de emprendedores para relanzar su utopía individualista.

Así como el PRO en tanto formación partidaria es fruto de la crisis de representación de los partidos argetninos tradicionales, el papel que cumplen los intelectuales en su interior - asumiendo los diversos perfiles que planteamosdebe considerarse a la luz de ese proceso y, más específicamente, en virtud de la pérdida de legitimidad que en el marco de la crisis de 2001-2002 experimentaron los expertos neoliberales formados fundamentalmente en el campo de las ciencias económicas, luego de haber vivido un período de verdadero ascenso al poder (Heredia). En los marcos del campo intelectual dicha crisis significó un reordenamiento a favor de fracciones que habían sostenido posturas antineoliberales y encarnaban trayectorias ligadas a diversas tradiciones del pensamiento crítico (Pulleiro). Mientras esas fracciones protagonizaron las disputas ideológicas más determinantes en los años siguientes y ocuparon los espacios centrales del campo cultural, otra franja de la intelectualidad participó de la experiencia política y cultural de consolidar una nueva fuerza política de derecha que irrumpió en medio de un proceso de reconfiguración de los compromisos políticos y se consolidó como respuesta a una sensibilidad caracterizada por una insatisfacción creciente, sobre todo, entre los sectores medios urbanos. El papel que esos intelectuales desempeñaron en la conformación del PRO, en las disputas ideológicas que se dieron en las diversas coyunturas que atravesó ese proceso y en la configuración de un modelo de intelectual de derechas moderno no puede ser ni exagerado ni subestimado. Esa indagación queda aún por hacerse en profundidad, el objetivo de este artículo estará cumplido si sus líneas básicas son retomadas para seguir desandando ese interrogante general. 


\section{REFERENCIAS BIBLIOGRÁFICAS}

Altamirano, Carlos. Notas para un programa de historia intelectual. Siglo XXI, 2005.

Altamirano, Carlos. Intelectuales: Notas de investigación. Norma, 2006.

Bourdieu, Pierre. Sobre la televisión. Anagrama, 1998.

Durán Barba, Jaime y Nieto, Santiago. Mujer, sexualidad, internet y política: Los nuevos electores latinoamericanos. Fondo de Cultura Económica, 2006.

Durán Barba, Jaime y Nieto, Santiago. El arte de ganar: cómo usar el ataque en campañas electorales exitosas. Debate, 2010.

Echt, Leandro. Los think tanks partidarios entre el conocimiento y la política: El caso de la Fundación Pensar y el PRO en Argentina. Tesis de Maestría Arts in Development Management and Policy, Georgetown University, 2016. https://doi.org/10.35537/10915/37436

Heredia, Mariana. Cuando los economistas alcanzaron el poder: (O cómo se gestó la confianza en los expertos). Siglo XXI, 2015.

Giordano, Verónica y Soler, Lorena. "Editoriales, ideas y nuevas derechas en América Latina". Ponencia: XIII Seminario Argentino Chileno, VI Seminario Cono Sur de Ciencias Sociales, Humanidades y Relaciones Internacionales, Universidad Nacional de Cuyo, 2016.

Giordano, Verónica. "Las ciencias sociales y los intelectuales en la perspectiva de las derechas latinoamericanas hoy". MILLCAYAC-Revista Digital de Ciencias Sociales, vol. 4, no. 7, 2017, pp. 165-180. https://doi. org/10.29340/11.1142

Mato, Daniel. "Think tanks, fundaciones y profesionales en la promoción de ideas (neo)liberales en América Latina". Cultura y neoliberalismo, compilador Alejandro Grimson, CLACSO, 2007, pp. 19-42.

Murillo, Susana. "Neoliberalismo y Estado de Excepción". Estado, gobierno $y$ gubernamentalidad. Neoliberalismo y Estado de excepción en Nuestramérica, compiladores Melisa Campana y José Giavedoni, Pegues, 2018, pp. 23-51. https://doi.org/10.24215/25251678e167

Natanson, José. ¿Por qué? La rápida agonía de la argentina kirchnerista y la brutal eficacia de una nueva derecha. Siglo XXI, 2018. https://doi. org/10.35305/tyd.v0i37.433

Laval, Christian y Dardot, Pierre. La nueva razón del mundo: Ensayo sobre la sociedad neoliberal. Gedisa, 2013.

Pecheux, Michel. Las verdades evidentes. Centro Cultural de la Cooperación, 2016. https://doi.org/10.22456/2594-8962.75694 
Pulleiro, Adrián. Liberales, populistas y heterodoxos: Estudios sobre intelectuales, cultura y política en la Argentina reciente. Batalla de Ideas, 2017.

Romé, Natalia. "Pensar en la coyuntura neoliberal". Política y subjetividad en la escena ideológica neoliberal, editoras Natalia Romé y Caroliina Collazo, Universidad de Buenos Aires, Facultad de Ciencias Sociales, 2018, pp. 4-22. https://doi.org/10.26422/aucom.2017.0602.ure

Rozitchner, Alejandro. "Ayudando a la desneurotización de la ciudadanía I". Estamos: Una invitación abierta, editores Alejandro Rozitchner y Marcos Peña, Planeta 2011, pp. 30-36.

Rozitchner, Alejandro. Ganas de vivir: La filosofia del entusiasmo. Sudamericana, 2012.

Rozitchner, Alejandro. La evolución de la Argentina. Mardulce, 2016.

Saferstein, Sebastián. La década editada: Los best sellers políticos y sus editores. Tesis de Doctorado, Facultad de Ciencias Sociales, Universidad de Buenos Aires, 2016. https://doi.org/10.26422/aucom.2017.0602.ure

Thompson, John B. Ideología y cultura moderna: Teoría crítica social en la era de la comunicación de masas. UAM, 1998.

Viotti, Nicolás. "Tu conflicto es un bajón". Revista Anfibia, marzo de 2018, http://www.revistaanfibia.com/ensayo/tu-conflicto-es-un-bajon/

Vommaro, Gabriel y Morresi, Sergio. "La ciudad nos une. La constitución del PRO en el espacio político argentino". Hagamos equipo. Pro y la construcción de la nueva derecha en Argentina, organizadores Gabriel Vommaro y Sergio Morresi. Ediciones UNGS, 2015, pp. 29-70. https:// doi.org/10.7440/colombiaint99.2019.04

Vommaro, Gabriel, et. al. Mundo PRO: Anatomía de un partido fabricado para ganar. Planeta, E-Book, 2015. https://doi.org/10.14409/es.v50i1.5953 